\title{
Les approches interculturelles dans l'éducation au Brésil
}

Intercultural trends in education in Brazil

Las tendencias interculturales en la educación brasileña

Mylene Santiago et Ana Canen

Traducteur : Jérôme Quintana

\section{OpenEdition}

1 Journals

Édition électronique

URL : http://journals.openedition.org/ries/3470

DOI : $10.4000 /$ ries.3470

ISSN : 2261-4265

Éditeur

Centre international d'études pédagogiques

\section{Édition imprimée}

Date de publication : 1 septembre 2013

Pagination : 43-54

ISBN : 978-2-85420-600-5

ISSN : $1254-4590$

Référence électronique

Mylene Santiago et Ana Canen, «Les approches interculturelles dans l'éducation au Brésil », Revue internationale d'éducation de Sèvres [En ligne], 63 | septembre 2013, mis en ligne le 01 septembre 2015, consulté le 01 mai 2019. URL : http://journals.openedition.org/ries/3470; DOI : 10.4000/ries.3470 


\section{Les approches interculturelles dans l'éducation au Brésil ${ }^{\star}$}

\section{Mylene Santiago Ana Canen}

La diversité culturelle est un sujet qui s'impose à toutes les sociétés contemporaines. L'intérêt croissant pour cette question va de pair avec les différents processus migratoires entre les pays et les régions, et avec l'émergence de conflits qui en résultent, face à la difficulté de faire cohabiter cette pluralité de cultures, de groupes ethniques, de religions et de visions du monde, dans le contexte social contemporain.

Le Brésil est un pays de grande diversité ethno-raciale. Sa population est composée de trois grands groupes: Indigènes, Blancs, Noirs. Les données issues du recensement ainsi que les données démographiques du Brésil relatives à la couleur (noire, brune, blanche, jaune ou indigène) sont obtenues par autodéclaration des personnes interrogées. La couleur brune est utilisée officiellement au Brésil pour catégoriser les personnes d'ascendance mixte. Il faut aussi prendre en considération la tendance à regrouper, à des fins d'enquête ou pour faciliter la formulation de politiques publiques, la population noire (preta) et brune (parda) dans la catégorie des Afro-Brésiliens ou de la population nègre (negra $)^{1}$.

La répartition de la population brésilienne selon la couleur ou l'appartenance raciale ci-dessous se conforme aux critères adoptés par l'Institut brésilien de la géographie et de la statistique (IBGE).

Répartition en pourcentage de la population du Brésil, par couleur ou appartenance raciale - 1999/2009

\begin{tabular}{|l|c|c|c|}
\hline Couleur ou appartenance raciale & $\mathbf{1 9 9 9}$ & $\mathbf{2 0 0 4}$ & $\mathbf{2 0 0 9}$ \\
\hline Noire (\%) & 5,4 & 5,9 & 6,9 \\
\hline Brune (\%) & 40 & 42,2 & 44,2 \\
\hline Blanche (\%) & 54 & 51,3 & 48,2 \\
\hline Jaune ou Indigène (\%) & 0,6 & 0,6 & 0,7 \\
\hline
\end{tabular}

Source : IBGE, Enquête nationale par échantillon de domiciles 1999/2009.

\footnotetext{
* Article traduit par Jérôme Quintana.

1. En portugais, preto et negro signifient tous deux « noir ». Le premier renvoie à la couleur à proprement parler, alors que le deuxième, sémantiquement plus large, englobe l'identité noire et le concept de négritude. Il est ainsi souvent préféré, d'autant que la distinction entre preto et pardo varie selon les régions, les ressentis personnels, etc. ( $N d T$ ).
} 
Les données de l'Enquête nationale par échantillon de domiciles (PNAD) indiquent une hausse de la population se déclarant noire ou brune, ces dix dernières années : leur pourcentage était respectivement de 5,4\% et $40 \%$ en 1999 , et de $6,9 \%$ et $44,2 \%$ en 2009 . Il est probable qu'il faille voir dans cette hausse une forme de récupération ou de renforcement de l'identité raciale à partir des politiques publiques orientées vers la réduction des inégalités raciales, comme par exemple les lois 10.639 et 11.645 qui instituent le caractère obligatoire de l'enseignement de «l'histoire et de la culture afro-brésilienne et indigène » dans le système scolaire.

Au Brésil, la thématique de la diversité culturelle est liée à l'histoire du refus de la reconnaissance de la diversité ethno-raciale de la population même du pays. Elle est également liée aux inégalités socioéconomiques qui découlent de ce processus et se manifestent à travers les emplois différenciés sur le marché du travail, la durée de scolarisation, l'accès à l'enseignement supérieur, les taux d'analphabétisme, les processus de mobilité sociale et de revenus des ménages par unité de consommation dans les différents groupes d'appartenance raciale. La figure ci-dessous, qui présente la répartition en pourcentage de la population selon la couleur ou l'appartenance raciale dans les principales régions du pays, indique une plus grande concentration de la population noire et brune dans les régions du Nord, du Nord-Est et du Centre-Ouest, considérées comme étant les régions les plus pauvres, comparativement aux régions du Sud-Est et du Sud.

\begin{tabular}{|l|c|c|c|c|}
\hline $\begin{array}{l}\text { Couleur ou } \\
\text { appartenance raciale }\end{array}$ & $\begin{array}{c}\text { Blanche } \\
(\%)\end{array}$ & $\begin{array}{c}\text { Noire } \\
(\%)\end{array}$ & $\begin{array}{c}\text { Brune } \\
(\%)\end{array}$ & $\begin{array}{c}\text { Jaune } \\
\text { ou indigène } \\
(\%)\end{array}$ \\
\hline Sud & 78,5 & 3,6 & 17,3 & 0,7 \\
\hline Sud-Est & 56,7 & 7,7 & 34,6 & 0,9 \\
\hline Nord & 23,6 & 4,7 & 71,2 & 0,4 \\
\hline Nord-Est & 28,8 & 8,1 & 62,7 & 0,3 \\
\hline Centre-Ouest & 41,7 & 6,7 & 50,6 & 0,9 \\
\hline
\end{tabular}

Source : IBGE, Enquête nationale par échantillon de domiciles, 2009.

Ce bref panorama du Brésil nous conduit, dans cet article, à analyser les tendances interculturelles de valorisation de la diversité ethnoculturelle dans le contexte des récentes politiques éducatives au Brésil, en nous intéressant tout particulièrement aux politiques liées à la formation des enseignants. Cette approche est pertinente dans la mesure où les enseignants, actuels et futurs, sont considérés comme étant des acteurs fondamentaux (et ce, dans le contexte de sociétés marquées par ces questions de pluralité et d'inégalité) pour préparer les générations futures à mieux apprécier la diversité et à mieux lutter contre les préjugés. 
La thèse que nous défendons ici est que les politiques éducatives de formation des enseignants au Brésil ont beaucoup progressé, dans leur formulation même, pour rendre l'intégration de la diversité culturelle plus concrète dans les programmes de formation des enseignants.

\section{FORMATION DES ENSEIGNANTS ET DIVERSITÉ CULTURELLE : LES TENDANCES}

Le multiculturalisme - ou les tendances interculturelles -, se réfère à un ensemble de réponses aux questions liées à la diversité culturelle, élaborées dans un contexte de politiques et de pratiques éducatives (Banks, 2004 ; Canen, 2009 ; Peters, 2005). Celles-ci visent à promouvoir la valorisation de la pluralité des identités individuelles, collectives (elles-mêmes représentées par des "groupes de collectivités » à partir de marqueurs tels que, entre autres, l'appartenance ethnique, raciale, le genre ou l'orientation sexuelle) et institutionnelles (ces dernières étant relatives aux cultures qui se développent dans les espaces institutionnels éducatifs). En élargissant le concept d'identité pour y inclure également les identités institutionnelles dans toute leur pluralité jusqu'aux organismes multiculturels (Canen \& Canen, 2005), le multiculturalisme sensibilise les différents acteurs à la singularité des cultures présentes dans les écoles, les établissements de formation des enseignants et les lieux d'exercice du pouvoir public.

À la fin des années 1980, et au début des années 1990, les divers débats en matière d'éducation et les politiques menées dans ce domaine ont donné un nouveau «souffle » à la formation des enseignants et à l'importance de la sensibilisation au thème de la diversité culturelle. Des directives curriculaires nationales furent définies pour les formations en pédagogie, par l'intermédiaire du rapport CNE/CP n 5 (2005), en soulignant l'idée que les étudiants en pédagogie devaient recevoir une formation pour garantir l'intégration pleine et entière des segments historiquement exclus de l'enseignement, dans le domaine des droits sociaux, culturels, économiques et politiques (p. 5).

À travers ces «directives", que l'on peut donc entendre comme étant des « orientations » en matière de curriculum, on remarque que la diversité est encore traitée de manière générale, sans formuler de recommandations - contrairement à ce qu'on pourrait attendre, selon des auteurs tels que Zeichner (2009) sur la façon d'inclure cette diversité dans les contenus curriculaires de la formation, ni sur les compétences à développer, ni même sur les critères d'évaluation à utiliser, ce qui relève de la responsabilité des institutions en charge de l'offre de formations en pédagogie. Toutefois, des perspectives multiculturelles semblent se profiler dans le document analysé.

Il convient de signaler également, dans le contexte de directives curriculaires prenant en compte les sensibilités multiculturelles, la promulgation des lois $10639 / 2003$ et $11645 / 08$, qui traitent de l'intégration des cultures indigènes 
et africaines et de celle des afro-descendants dans le curriculum scolaire. Ces lois ont par ailleurs permis l'élaboration de directives curriculaires nationales pour l'éducation aux rapports ethno-raciaux et pour l'enseignement de l'histoire et de la culture afro-brésilienne et africaine (MEC, 2005). Ces directives permettent de prendre conscience des questions liées à l'inégalité, à la marginalisation, aux préjugés à l'encontre des identités collectives noire et indigène, même si les documents officiels semblent ne pas éviter certaines perspectives dichotomiques qui figent les rapports entre Noirs et Blancs, ignorant les processus d'hybridation et les mouvements de construction et de reconstruction identitaire.

Il faut rappeler que les politiques de formation initiale et continue des enseignants ont fait l'objet d'une attention particulière avec le décret 6755/09 (MEC, 2009) qui a institué la " Politique nationale de formation des personnels de l'enseignement de base ${ }^{2}$ » (CAPES, 2010), laquelle vise à organiser la coopération entre différentes entités (fédérale, étatique et municipale) afin de proposer une offre de formation initiale et continue des enseignants de l'école publique, sous la responsabilité de la Coordination de perfectionnement du personnel de l'enseignement supérieur (CAPES).

Cependant, comme nous allons le voir, le secrétariat chargé de la formation continue, de l'alphabétisation et de la diversité au ministère de l'Éducation (MEC) a actuellement pour mission de mettre en ouvre les politiques publiques éducatives en les articulant aux systèmes d'enseignement et en veillant à ce qu'elles traitent des questions liées à la diversité culturelle dans le contexte brésilien.

\section{LA DIVERSITÉ CULTURELLE : UN PRINCIPE DIRECTEUR DES POLITIQUES PUBLIQUES ÉDUCATIVES ?}

Le secrétariat chargé de la formation continue, de l'alphabétisation et de la diversité au ministère de l'Éducation (Secad\MEC) a été créé par le gouvernement fédéral en 2003, avec pour mission d'institutionnaliser dans le système national d'enseignement la reconnaissance de la diversité socioculturelle, en la posant comme un principe dans l'élaboration de la politique publique éducative, et en mettant en évidence le rapport entre l'inégalité de l'accès à l'éducation et le taux de réussite ou de poursuite des études, dans un contexte historique d'exclusion où on a dévalorisé et refusé de prendre en compte les différences ethno-raciales et culturelles, mais aussi celles liées à l'identité sexuelle et au genre, dans les écoles au Brésil (Secad, MEC, 2007).

2. Au Brésil, l'enseignement dit « de base » (educação básica) se répartit en trois étapes qui couvrent toute la formation, depuis le pré-primaire jusqu'au secondaire. $(\mathrm{NdT})$ 
La Secad se divise en cinq départements : le département chargé de la politique en matière d'éducation spécialisée ; le département chargé de la politique éducative en milieu rural, en direction des populations indigènes, visant à faciliter les rapports ethno-raciaux; le département chargé de la politique éducative en matière de droits de l'homme et de citoyenneté ; le département chargé de la politique d'alphabétisation et d'éducation des jeunes et des adultes ; enfin, le département chargé de la politique éducative en direction de la jeunesse.

Chaque département développe un ensemble de programmes et le portail officiel du ministère de l'Éducation met à disposition une série de publications sur les programmes et les actions qu'il entreprend. Afin d'éclairer notre analyse, et étant donné l'accent mis actuellement sur les politiques ethnoraciales, nous allons présenter certaines actions entreprises par le département chargé de la politique éducative en milieu rural, en direction des populations indigènes, visant à faciliter les rapports ethno-raciaux.

\section{L'éducation interculturelle en faveur des populations indigènes}

L'analyse du document "Educação Escolar Indígena : diversidade sociocultural indígena ressignificando a escola ${ }^{3} »$ (2007) met en évidence le fait que les politiques publiques en matière d'enseignement scolaire en faveur des populations indigènes, dans la période qui a suivi la Constitution de 1988, sont guidées par le respect du savoir, des traditions et des coutumes de chacune des communautés qui, rassemblant plus de 225 peuples, constituent la diversité du pays. De fait, les divers programmes et actions reflètent l'intention suivante: valoriser et maintenir la sociodiversité indigène et, en d'autres termes, référencer les principes et les concepts utilisés dans les projets relatifs à la société et à l'identité, élaborés de façon autonome par chaque peuple indigène.

Le document met en évidence l'inadéquation des politiques et actions qui considèrent les peuples indigènes sans établir de distinction, sans examiner leurs spécificités sur un plan culturel et linguistique. Il souligne également le fait que, pour tenir compte des perspectives et des revendications socio-environnementales de chaque peuple, il convient de relever le défi consistant à déterminer les priorités en termes d'orientation politique en accord avec les enseignants, les représentants de chaque peuple et les gestionnaires publics.

Parmi les fondements de l'enseignement scolaire en faveur des populations indigènes figurent les pédagogies indigènes. Celles-ci apparaissent de manière évidente dans les choix méthodologiques opérés pour l'acquisition 
de la lecture et de l'écriture, dans l'utilisation du temps et de l'espace scolaire, dans le regroupement des élèves et dans les diverses activités réalisées à partir du lien entre école et vie de la communauté (PIANTA, 2003, Secad \MEC, 2007).

La pédagogie indigène s'efforce de mettre en valeur et d'entretenir la sociodiversité indigène, en prenant pour référence les principes et les concepts utilisés dans les projets sociaux et identitaires élaborés de manière autonome par chaque peuple indigène. Parmi les principaux concepts liés à l'éducation scolaire indigène, citons la territorialité, qui implique d'une part la reconnaissance de l'organisation sociale des peuples indigènes et du rapport entre école et durabilité socio-environnementale, et qui englobe d'autre part l'articulation entre savoirs traditionnels et nouvelles technologies afin que ces peuples puissent développer la gestion de leurs territoires de manière autonome, à partir de leurs propres besoins et intérêts.

C’est parmi les idées qui se sont développées à partir de la réflexion et de l'action suscitées par les expériences de rénovation des pratiques pédagogiques et curriculaires de l'école indigène, que nous situons les questions de multiethnicité et de pluralité, dont la compréhension est indispensable pour la formulation de politiques et d'actions appropriées aux réalités de chaque peuple indigène.

En présentant l'interculturalité comme caractéristique première de l'école indigène, le document formule les prescriptions suivantes :

L'interculturalité prend en compte la diversité culturelle dans le processus d'enseignement et d'apprentissage. L'école doit s'appuyer sur les valeurs, les savoirs traditionnels et les pratiques de chaque communauté, et garantir l'accès aux savoirs et aux technologies utiles propres à la société nationale, pour faciliter le processus d'interaction et de participation citoyenne au sein de la société nationale. De ce fait, les activités curriculaires doivent être pertinentes et replacées dans le contexte du vécu des apprenants et de leurs diverses communautés d'appartenance.

Cette définition de l'interculturalité nous semble relever de la simple prescription, dans la mesure où le document ne mentionne aucunement la façon de mettre en application ces exigences interculturelles dans le cadre du processus éducatif en faveur des populations indigènes, notamment en ce qui concerne la garantie de l'accès aux savoirs et aux technologies de la société nationale. Les droits linguistiques sont également mis en avant dans le processus interculturel, tout comme le "devoir " qui incombe aux enseignants indigènes de participer aux stages de formation continue susceptibles de renforcer et de valoriser la langue indigène comme langue d'enseignement et de communication.

Le document admet l'existence d'impasses dans la mise en application des principes d'interculturalité et de bilinguisme dans l'enseignement scolaire en faveur des populations indigènes, principes garantis dans divers textes juridiques et normatifs. 
Face à de tels défis, il est aujourd'hui admis qu'on procède au mieux à des adaptations qui demeurent peu conformes aux perspectives de l'enseignement scolaire en faveur des populations indigènes et peu satisfaisantes également pour les différents acteurs issus de ces populations. Afin de répondre à cet état de fait, le Secad a mis en œuvre des programmes visant à garantir le droit des indigènes à l'éducation de base interculturelle en matière de formation d'enseignants indigènes et de publication de supports didactiques bilingues rédigés par des auteurs indigènes. Ces programmes s’inscrivent parmi d'autres actions visant à promouvoir la participation indigène ainsi que l'accompagnement et l'évaluation des politiques éducatives du ministère de l'Éducation.

Au Brésil, l'approche de l'interculturalité indigène s'est concentrée exclusivement sur les communautés homogènes, situées en territoire indigène. Sans sous-estimer l'importance que revêt cette tendance, notons toutefois que certains indigènes ne vivent pas sur leur terre d'origine et que de nombreux Brésiliens ont des origines indigènes. Il nous semble donc nécessaire que les orientations et les programmes du ministère de l'Éducation s'étendent aux indigènes insérés dans l'ensemble de la société brésilienne.

\section{Les défis relatifs à l'entrée en application de la loi 10.639/2003}

La loi 10.639/2003 a rendu obligatoire l'enseignement de l'histoire et de la culture afro-brésiliennes dans l'éducation de base. Cette loi a été instituée grâce à la résolution $\mathrm{n}^{\circ} 1$ du 17 juin 2004 adoptée par le Conseil national de l'éducation, qui a émis les directives curriculaires nationales en matière d'éducation aux rapports ethno-raciaux et d'enseignement de l'histoire et de la culture afro-brésiliennes et africaines.

Partant du constat que les intentions prévues par la loi et les directives ne suffisaient pas pour mettre en œuvre des pratiques curriculaires dans le quotidien de l'école, un atelier de travail a été mis en place à Brasilia en 2007, à l'initiative de l'UNESCO et du Secad, regroupant quarante chercheurs et militants s'intéressant de près aux rapports ethno-raciaux dans le domaine de l'éducation. Ces derniers ont cherché à déterminer où en était la mise en application de la loi 10.639/2003. En fin de séance, un groupe de travail a été créé afin de fixer des objectifs, de proposer des stratégies et de définir un certain nombre d'indicateurs nationaux pour la mise en application et l'accompagnement de cette loi.

Le travail réalisé par ce groupe a débouché sur la "Proposition d'un plan national de mise en œuvre des directives curriculaires nationales en matière d'éducation aux rapports ethno-raciaux, favorisant l'enseignement de l'histoire et de la culture afro-brésiliennes et africaines - Loi 10.639/2003». 
Ce document retrace l'historique du processus de discrimination raciale qui a reposé, dans un premier temps, sur l'idéologie du blanchiment physique et culturel de la nation via l'immigration européenne et, dans un deuxième temps, sur le mythe de la démocratie raciale, fondateur du discours sur l'unité entre blancs, indiens et noirs, lequel a contribué à dissimuler les hiérarchies et les discriminations constitutives des rapports entre blancs et non blancs (Secad/ MEC, 2008).

Selon le document en question, la loi 10.639 constitue un repère historique important dans ce qui a conduit au besoin de modifier radicalement la structure curriculaire des formations qui, à tous les niveaux, à toutes les étapes et dans toutes les modalités de l'enseignement, ignoraient ou passaient sous silence la contribution africaine et afro-brésilienne à l'élaboration des connaissances dans les différents domaines du savoir. Outre les modifications apportées au curriculum, il s'est également agi de répondre au problème soulevé par certaines images présentes dans les manuels scolaires, mais également certains termes péjoratifs utilisés dans les supports didactiques, voire dans les contenus proposés lors des formations à destination des enseignants. En tant que politique éducative, le principal objectif de cette loi était, d'une part, de rompre avec un modèle de posture pédagogique qui ne reconnaissait pas les différences induites par notre processus de formation nationale et, d'autre part, de promouvoir la lutte stratégique contre les cultures et les pratiques racistes et discriminatoires, institutionnalisées dans le quotidien des écoles et dans les systèmes d'enseignement.

Le document souligne le fait que, même s'il existe des expériences très intéressantes axées sur l'éducation aux rapports ethno-raciaux, celles-ci se limitent pour la plupart du temps à l'action isolée de professionnels engagés dans la défense de valeurs prônant l'égalité raciale. Cela débouche en conséquence sur des projets qui ne s'inscrivent pas dans la continuité et qui ne s'articulent pas aux politiques curriculaires de formation des enseignants. Pour parer à ces difficultés, des axes stratégiques sont proposés : renforcement du cadre légal; politique de formation à destination des gestionnaires et des professionnels de l'éducation; politique de développement du matériel didactique; gestion démocratique et mécanismes de participation sociale ; conditions institutionnelles (financement, sensibilisation et communication, recherche, équipes et mode de collaboration); enfin, évaluation et suivi.

On notera par ailleurs que les politiques publiques éducatives ont traité la diversité indigène et afro-brésilienne de manière différenciée, afin de lutter contre les inégalités ethno-raciales présentes dans le système scolaire brésilien aux différents échelons de l'enseignement. Ainsi, le ministère de l'Éducation a favorisé la formation d'enseignants indigènes dans le cadre de cursus interculturels, avec le soutien de certaines universités publiques - Université fédérale de Roraima (UFRR), Université de l'État du Mato Grosso (Unemat), Université 
fédérale de Minas Gerais (UFMG), Université de l'État d'Amazonas (UEA) - et de partenariats établis avec les secrétariats d'État chargés de l'éducation ainsi que la Fondation nationale de l'Indien (Funai). Un constat: les politiques éducatives en faveur des populations indigènes, tout autant que celles relatives à l'application de la loi 10.639/2003, mettent l'accent, entre autres, sur le processus de formation des enseignants comme axe stratégique.

Akkari et Santiago (2010) ont observé que, malgré l'existence de lois et de processus de normalisation favorisant l'intégration du débat autour des questions ethno-raciales dans le contexte scolaire, le système d'enseignement brésilien est insuffisamment préparé pour faire face à une perspective interculturelle. Ils confirment ainsi l'importance de former des enseignants possédant une orientation interculturelle, engagés dans cette perspective et conscients de la nécessité de promouvoir un enseignement culturellement sensible, qui tienne compte des perspectives des élèves issus de divers groupes culturels, avec entre autres différentes identités de genre et de race et différents modèles linguistiques. Face à ce contexte, nous allons présenter les tendances interculturelles telles qu'elles sont déclinées dans le Plan national de l'éducation pour la décennie 2011-2020.

\section{Perspectives futures : LES POSSIBILITÉS INTERCULTURELLES}

La Conférence nationale de l'éducation (CONAE) qui s'est tenue du 28 mars au $1^{\text {er }}$ avril 2010 portait sur l'objectif suivant : "CONAE : l'élaboration du système national articulé ; plan national d'éducation, directives et stratégies d'action ». En termes de sensibilité à la diversité culturelle, point central de cet article, on constate que le document, dès la présentation, affirme proposer des « directives, objectifs et actions en faveur de la politique nationale d'éducation, dans une perspective d'intégration, d'égalité et de diversité (nos italiques), ce qui constitue un repère historique pour le système éducatif brésilien, qui s'inscrit dans la modernité » (ministère de l'Éducation, document final de la CONAE, mai 2010).

Le document, s'appuyant sur un précédent document de base, est le fruit de longs débats. Il avait pour principal objectif d'encourager de nouvelles perspectives pour le Plan national d'éducation 2011-2020, avec à l'horizon un Système national d'éducation au Brésil, défini comme étant un pacte fédérateur pour le nouveau plan national de l'éducation susmentionné. Il se divise en six axes : rôle de l'État et garantie du droit à une éducation de qualité (...); qualité de l'éducation, gestion démocratique et évaluation; démocratisation de l'accès, taux de réussite et de poursuite des études ; formation et valorisation des professionnels de l'éducation; financement de l'éducation et contrôle social ; enfin, justice sociale, éducation et travail : intégration, diversité et égalité. 
Une première série d'observations concerne la séparation entre les différents axes : la formation des enseignants, d'une part, et l'intégration, la diversité et l'égalité, d'autre part. Nous constatons également une séparation entre les intentions, telles que nous les avons signalées plus haut, et les débats plus spécifiques sur le curriculum scolaire et sur celui relatif à la formation des enseignants et à la gestion même de l'éducation. Par exemple, lorsqu'on analyse l'axe $n^{\circ} 6$ de ce document, on s'aperçoit qu'il semble se concentrer essentiellement sur le débat autour d'actions de lutte contre la discrimination et autour des droits de l'homme, sans relier ces thématiques à l'axe portant sur la formation des enseignants et sur le curriculum scolaire et pédagogique.

Une deuxième série de considérations traite de l'explicitation claire de la sensibilité multiculturelle, sur le plan des intentions, telle qu'elle s'exprime dans le document. En termes de conception curriculaire et du rapport de celle-ci à la diversité culturelle, on constate, par exemple, que l'accent est mis sur l'élaboration d'une "base commune nationale afin de garantir une formation de base commune, ainsi que le respect des valeurs culturelles et artistiques, tant nationales que régionales », conformément à l'article 210 de la Constitution fédérale. Une telle perspective intègre le respect de la diversité culturelle et de la perspective multiculturelle dans le cadre de l'état-nation démocratique, pour reprendre l'idée exprimée par des auteurs tels que Banks (2004), en évitant tout relativisme culturel et en supposant un dialogue entre diversité et perspectives universalistes issues des directives nationales. On souligne l'importance de la transversalité de l'éducation spécialisée dans l'enseignement et on insiste sur le fait que l'objectif est de transformer les systèmes éducatifs en

«systèmes inclusifs, qui prennent en compte la diversité dans un but d'égalité, en garantissant le droit à l'égalité et à la diversité ethno-raciale, à la diversité de genre, d'âge, d'orientation sexuelle et de religion, et en garantissant également leurs droits aux élèves handicapés, ou atteints de troubles envahissants du développement, ou encore présentant de fortes aptitudes / surdoués ».

Nous constatons que, par rapport aux précédents documents législatifs, l'éventail des identités collectives présentes dans celui-ci s'élargit. On dépasse la question de l'identité collective raciale et l'on perçoit, dans un horizon défini par le document, les potentiels multiculturels aujourd'hui présents.

Ainsi, l'analyse des documents législatifs permet d'entrevoir des perspectives positives, d'un point de vue multiculturel, même s'il révèle, par ailleurs, une certaine persistance à considérer la diversité culturelle comme étant un sujet distinct des thèmes curriculaires plus traditionnels à l'école et dans la formation des enseignants. Les orientations futures pourraient avoir pour objectif d'approfondir les diverses manières d'intégrer les sensibilités interculturelles au curriculum spécifique de la formation des enseignants, pour reprendre une proposition faite par Banks (2004), Canen (2009) et Zeichner (2009a), de sorte que, dans la pratique, les formations à destination des enseignants puissent effectivement 
constituer des instances de valorisation de la diversité, favorisant le développement d'espaces institutionnels qui soient multiculturels dans les faits (Canen \& Canen, 2005).

Cet aspect mériterait de faire l'objet de plus amples recherches, afin de combler ce manque et de contribuer ainsi à l'expansion des recherches multiculturelles, en tentant d'articuler celles-ci au curriculum prescrit et au curriculum enseigné.

La thèse défendue dans cet article repose sur l'idée qu'il est important de prendre en compte la diversité culturelle et de l'incorporer aux politiques éducatives. Nous avons ainsi abordé les politiques en matière de formation des enseignants au Brésil comme étude de cas. Nous avons par ailleurs montré que le souci de promouvoir la diversité culturelle, dans le cadre des politiques curriculaires nationales, apparaît de manière plus évidente dans les parcours de formation spécifiques, axés sur les identités collectives, par exemple les populations indigènes, noires et quilombolas ${ }^{4}$, sans que cette préoccupation, au demeurant valable et pertinente, ne soit reliée au curriculum scolaire ni à celui portant sur la formation des enseignants.

Étant donné la spécificité brésilienne en matière de traitement de la diversité culturelle à l'école, ce thème doit figurer au centre des formations proposées. En effet, le "noyau structurant " du système éducatif repose sur un modèle unique qui résiste à l'idée de se libérer, entre autres, de hiérarchies sociales, raciales, culturelles et de genre.

Pour que l'impact sur les pratiques enseignantes soit plus grand, il nous paraît encore nécessaire, en termes de perspectives, de favoriser un certain nombre de points. Par exemple, éviter de mettre l'accent de manière excessive sur les identités collectives en tant que balises de formulation des directives en matière de diversité, tout en intégrant par ailleurs les sensibilités multiculturelles aux disciplines, domaines, aptitudes et compétences développés dans le curriculum scolaire et dans celui relatif à la formation des enseignants; améliorer la formation continue des enseignants, en mettant l'accent sur la sensibilité à la diversité ; promouvoir les stages de formation continue axés sur la pluralité des expériences dans le domaine de la diversité à l'école, en transformant les enseignants et les gestionnaires en chercheurs de terrain, plutôt qu'en récepteurs de programmes préétablis.

La réflexion menée ici à partir des données analysées nous permet d'affirmer que les politiques évoquées ont progressé de manière positive en termes de sensibilité à la diversité culturelle, dans les orientations données au

4. Les quilombolas sont les descendants d'esclaves en fuite initialement regroupés dans les quilombos, colonies situées au fond de forêts inaccessibles. Avec la fin de l'esclavage au Brésil en 1888, les quilombos ont disparu, mais certains des descendants ont continué à occuper les terrains, et ce jusqu'à ce jour. (NdT) 
curriculum relatif à la formation des enseignants. Les indications données plus haut constituent des possibilités pour accroître la sensibilité à la diversité culturelle et laissent entrevoir des perspectives plus concrètes de traduction de ces présupposés en matière de curriculum prescrit et de curriculum enseigné.

\section{BIBLIOGRAPHIE}

AKKARI A., SANTIAGO M. (2010) : A gestão da diversidade cultural no contexto educacional brasileiro. Revista Educação em Questão, Natal, v. 38, n 24, mai/août 2010, p. 9-33.

BANKS J. A. (Ed.) (2004) : Diversity and Citizenship Education: global perspectives. San Francisco, USA : Jossey-Bass Eds, 2004.

Brasil MEC (2005) : Diretrizes Curriculares Nacionais para a Educação das Relações Étnico-Raciais e para o Ensino de História e Cultura Afro-Brasileira e Africana. http://www.uel.br/projetos/leafro/pages/arquivos

Brasil MEC (2008) : Proposta de Plano Nacional de Implementação das Diretrizes Curriculares Nacionais da Educação das Relações Étnico-raciais e para o Ensino de História e Cultura Afro-Brasileira e Africana - Lei 10.639/2003. http://portal.mec.gov.br/

Brasil MEC (2006) : Diretrizes Curriculares Nacionais para o Curso de Pedagogia. http://portal.mec.gov.br/

Brésil MEC (2010) : Documento Final da CONAE, http://conae.mec.gov.br, 27/05/2010.

Brasil, Secad/MEC (2007): Educação Escolar Indígena: diversidade sociocultural indígena ressignificando a escola. Brasília : DF. http://portal.mec.gov.br/

Brasil, Presidência da República (2003) : Lei 10639/03.

Brasil, Presidência da República (2008) : Lei 11645/08.

Brasil, CAPES (2010) : Política Nacional de Formação de Profissionais do Magistério da Educação Básica. Brasília, CAPES, DOU, 30 janvier 2010, nº 21, http://www.capes. gov.br/.

CANEN A. (2009): Teacher Education and Competence in an Intercultural Perspective. Koln : Lambert Academic Publishing AG \& Co, 2009. 386 p.

CANEN A. G. \& CANEN A. (2005) : Organizações Multiculturais. Rio de Janeiro : Ed. Ciência Moderna.

CANEN A. \& OLIVEIRA A.M.A. (2002) : Multiculturalismo e currículo em ação : um estudo de caso. Revista Brasileira de Educação. Set/dez., n 21. http://www.scielo.br/ pdf/rbedu/n21/n21a05.pdf/.

PETERS M. (2005) : Education, Post-Structuralism and the Politics of Difference. In : Canen A. \& Peters M. (Eds), Issues and Dilemmas of Multicultural Education: theories, policies and practices. Policy Futures in Education, vol. 3, n 4, 2005, p. 436-445.

ZEICHNER K. M. (2009): Teacher Education and the Struggle for Social Justice. London : Routledge. 